\title{
The classification of tetraploid wheat by phylogenetic and cytogenetic analyses
}

\author{
S.-Y. LI ${ }^{1,2}$, L. XI ${ }^{1,2}$, H.-J. LIU ${ }^{1,2}$, W. ZHU ${ }^{1}$, L.-L XU1 ${ }^{1}$, Y. WANG ${ }^{1,2}$, J. ZENG ${ }^{3}$, X. FAN ${ }^{1,2}$, L.-N. SHA ${ }^{1,2}$, \\ H.-Q. ZHANG ${ }^{1,2}$, W.-L. QI ${ }^{4}$, G.-Y. CHEN ${ }^{1,2}$, Y.-H. ZHOU ${ }^{1,2}$, and H.-Y. KANG ${ }^{1,2 *}$ \\ State Key Laboratory of Crop Gene Exploration and Utilization in Southwest China, Sichuan Agricultural \\ University, Chengdu 611130, Sichuan, P.R. China ${ }^{1}$ \\ Triticeae Research Institute, Sichuan Agricultural University, Chengdu 611130, Sichuan, P.R. China ${ }^{2}$ \\ College of Resources, Sichuan Agricultural University, Chengdu 611130, Sichuan, P.R. China ${ }^{3}$ \\ College of Chemistry and Life science, Chengdu Normal University, Chengdu 611130, Sichuan, P.R. China ${ }^{4}$
}

\begin{abstract}
Tetraploid wheat (Triticum turgidum L.) is an important species within the genus Triticum and harbors many desirable agronomic traits. The classification, origin, and evolution of tetraploid wheat remain confused and controversial, resulting in useless germplasm resources. Two classification systems for tetraploid wheat are widely used: 1) tetraploid wheat comprises two species; 2) all forms of tetraploid wheat are classified as one species. The present study aimed to reassess the classification of tetraploid wheat using phylogenetic analysis of nuclear rDNA internal transcribed spacer region (ITS) sequence data, fluorescence in situ hybridization (FISH) karyotyping, and observation of meiotic pairing behavior in F $_{1}$ hybrids. Network analysis of ITS sequences indicates that tetraploid wheat was not closely related to other Triticeae species with the exception of Aegilops speltoides and Ae. sharonensis. Phylogenetic analysis of ITS sequences and FISH show that Triticum turgidum and T. timopheevii clustered on distinct branches, and meiotic pairing in $\mathrm{F}_{1}$ hybrids of these species showed a high frequency of univalents. Meiotic behavior of $F_{1}$ hybrids among forms of $T$. turgidum revealed a low number of univalents (means $<2$ ) except for T. turgidum ssp. dicoccoides. The significant variation on chromosomes $1 \mathrm{~A}, 2 \mathrm{~A}, 5 \mathrm{~A}$, $1 \mathrm{~B}, 2 \mathrm{~B}, 3 \mathrm{~B}$, and $6 \mathrm{~B}$ in the FISH hybridization patterns were observed between T. turgidum ssp. dicoccoides and other T. turgidum accessions. Furthermore, the results of ITS phylogenetic analyses correspond closely with observations of meiotic behavior and FISH karyotyping. The present results indicate that T. turgidum and T. timopheevii are two distantly related species of different origins. Triticum turgidum ssp. dicoccoides should be maintained as a subspecies of T. turgidum whereas other forms of T. turgidum should be reclassified as varieties.
\end{abstract}

Additional key words: chromosome pairing behavior, FISH analysis, ITS, Triticum turgidum.

\section{Introduction}

Tetraploid wheat (Triticum turgidum L.) has played an important role in the history of human civilization. As relatives of Triticum aestivum L., tetraploid wheat possesses many desirable agronomic traits and thus can be used as parents in breeding programs to improve the yield and quality of T. aestivum (Zaharieva et al. 2010). With hard kernel texture and abundant protein for pasta, T. turgidum ssp. durum has become the second cultivated wheat after T. aestivum (Oliveira et al. 2012). Triticum turgidum ssp. durum was crossed with Thinopyrum elongatum (Host) A. Löve, and a series of addition lines and substitution lines were successfully raised as breeding material to enhance the Fusarium head blight resistance of wheat cultivars (Jauhar et al. 2008, 2009, 2014, Forte et al. 2014, Kuzmanović et al. 2014). Many valuable genes have been identified in T. turgidum ssp. dicoccoides (Körn. ex Aschers. et Graebn. Thell.), including the stripe rust resistance genes $\mathrm{Yr} 15$ and $\mathrm{YrH}$ 52, the powdery mildew resistance genes $P m 16, P m 26$, and $P m 30$, and the high grain protein content gene $G P C-B 1$, which are widely used in breeding for high yield (Gerechmr-Amitai et al. 1989, Reader and Miller 1991, Peng et al. 1999, Rong et al.

\footnotetext{
Submitted 16 October 2019, last revision 18 January 2020, accepted 23 January 2020.

Abbreviations: AFLP - amplified fragment length polymorphism; DAPI - 4-6-diamino-2-phenylindole; FISH - fluorescence in situ hybridization; ITS - internal transcribed spacer region; MJ - median-joining; NJ - neighbor-joining; PMC - pollen mother cell.

Acknowledgements: We thank Dr. Lianquan Zhang, Sichuan Agricultural University, Chengdu, China, for kindly supplying the tetraploid wheat materials used in this study. This work was funded by the National Key Research and Development Program of China (Nos. 2016YFD0102000 and 2017YFD0100905) and the National Natural Science Foundation of China (Nos. 31771781 and 31971883 ).

* Corresponding author; e-mail: houyang.kang@sicau.edu.cn
} 
2000, Liu et al. 2002, Chen et al. 2005, Uauy et al. 2006, Peleg et al. 2008). Other forms of tetraploid wheat are also excellent genetic resources for precocity, pest resistance, lodging resistance, salt tolerance, or nutrient abundance (Oliveira et al. 2012).

For effective use of germplasm resources, a strictly defined classification of tetraploid wheat is crucial (Goncharov et al. 2009). Taxonomists have proposed different classification systems based on morphological, cytological, or genetic characteristics, and consequently the determination of species and subspecies of tetraploid wheat is extremely confusing and controversial (Goncharov et al. 2011). Bowden (1959) treated allotetraploid wheat as one species, T. turgidum, with three varieties, two variants, and eight cultivars. Morris and Sears (1967) recognized T. timopheevii as a species based on cytogenetic evidence and geographic distribution. Two major classification systems for tetraploid wheat have been proposed: 1) MacKey (1966) and Van Slageren (1994) classified all tetraploid wheat as subspecies, MacKey (1975) further classified durum and turanicum ssp. as cultivars of T. turgidum; 2) on the basis of morphological observations, Dorofeev et al. (1979) concluded that all ssp. of tetraploid wheat are individual species.

Given genetic and morphological diversity, the evolution of tetraploid wheat under domestication has not been widely reported (Matsuoka et al. 2011). Domesticated Triticum turgidum (emmer wheat) is suggested to have originated from $T$. turgidum ssp. dicoccoides in southeastern Turkey (Özkan et al. 2011). Genetic and archaeological evidence indicate that following the initial domestication of $T$. turgidum ssp. dicoccoides, naked tetraploid wheats evolved from emmer wheat (Salamini et al. 2002, Özkan et al. 2005). Using cluster analysis of amplified fragment length polymorphism (AFLP), allele frequencies revealed that all hulled wheats are clustered in one group, and all types of naked wheat are included in a distinct group (Salamini et al. 2002). $Q$ gene sequences indicate that tetraploid wheat can be divided into two clades supported by spike morphological traits, namely non-free-threshing, fragile, and free-threshing, non-fragile, normal wheats (Sormacheva et al. 2015). On the basis of genetic diversity indicated by four sets of genetic markers, Oliveira et al. (2012) emphasized that T. turgidum ssp. durum and T. turgidum ssp. turgidum share a common gene pool and comprise a genetically different population from T. turgidum ssp. dicoccon and T. turgidum ssp. dicoccoides. Phylogenetic analysis of four single-copy nuclear gene showed that each clade contained naked tetraploid wheat and hulled tetraploid wheat, which is indicative of a close evolutionary relationship between these two forms of tetraploid wheat (Takenaka et al. 2010). Haplotype analysis of sequence data for the photoperiod-related $P p d-A 1$ and $P p d-B 1$ genes in tetraploid wheat indicated that gene flow between hulled and naked tetraploid wheat had occurred (Takenaka et al. 2012). Phylogenetic reconstruction of $P p d-A 1 b$ haplotypes revealed that T. turgidum ssp. turgidum and T. turgidum ssp. ispahanicum were not clustered together with T. turgidum ssp. dicoccoides, T. turgidum ssp. durum, T. turgidum ssp. dicoccon, and T. turgidum ssp. carthlicum were closely related (Muterko et al. 2015). Thus, despite considerable research, the phylogenetic history of tetraploid wheat remains contentious.

Our previous study indicated that the hulled tetraploid wheat accessions formed a subclade, and naked tetraploid wheat got other subclade, and at least two intermediary subspecies were involved in the evolution of T. turgidum (Tang et al. 2017). In this study, we further sequenced and analyzed the nuclear rDNA internal transcribed spacer region (ITS) sequences, observed chromosome pairing in artificial $F_{1}$ hybrids, and assessed the chromosomal distribution of signal sites for the fluorescence in situ hybridization (FISH) probes pTa535 and pSc119.2 to reevaluate the phylogenetic relationships and taxonomic classification of tetraploid wheat.

\section{Materials and methods}

Plant materials: Eleven taxa of tetraploid wheat were used for the ITS sequence analysis. The genome composition, provenance, and GenBank accession numbers for samples included in the study are listed in Table 1 Suppl. The ITS sequences with $\mathrm{MH}$ numbers of tetraploid wheat are newly reported in this study. The sequences for related species were downloaded from the GenBank database. Bromus inermis was used as outgroup. A collection of 21 tetraploid wheat accessions including 11 taxa were employed for FISH (Table 1 Suppl.). The $F_{1}$ hybrids derived from 14 artificial crosses between tetraploid wheat accessions were used to study meiotic pairing behavior (Table 1). The accessions designated by PI and Citr numbers were kindly provided by American National Plant Germplasm System (Pullman, USA), and the accessions with AS numbers were collected by Triticeae Research Institute, Sichuan Agricultural University, China.

Phylogenetic analysis: Total genomic DNA was extracted from fresh leaf tissue using the standard cetyltrimethylammonium bromide extraction protocol (Doyle and Doyle 1990). Primers ITS-4 (5'-TCCTCCGCTTATTGATATGC-3') and ITS-L (5'-TCGTAACA AGGTTTCCGTAGGTG-3') were used to amplify the entire ITS region (Hsiao et al. 1995). The PCR reaction was accomplished in a $50 \mathrm{~mm}^{3}$ volume containing $5 \mathrm{~mm}^{3}$ of $10 \times$ Taq polymerase buffer, $1.5 \mathrm{mM}$ of each primer, $10 \mathrm{mM}$ of dNTP mix, $20 \mathrm{ng}$ of template DNA, $2.5 \mathrm{mM} \mathrm{MgCl}_{2}$, and $2 \mathrm{U}$ high-fidelity ExTaq ${ }^{\circledR}$ DNA polymerase (Takara, Dalian, China). A GeneAmp ${ }^{\circledR}$ 9700 thermal cycler (Applied Biosystems, Foster City, CA, USA) was used to perform the PCR reaction. The amplification procedure consisted of pre-denaturation at $94{ }^{\circ} \mathrm{C}$ for $5 \mathrm{~min}, 35$ cycles at $94{ }^{\circ} \mathrm{C}$ for $1 \mathrm{~min}$, at $52{ }^{\circ} \mathrm{C}$ for $1 \mathrm{~min}$, and at $72^{\circ} \mathrm{C}$ for $1 \mathrm{~min}$, followed by $72{ }^{\circ} \mathrm{C}$ for $7 \mathrm{~min}$. The PCR products were cloned into the pMD19-T vector (Takara) by TA cloning. For recovery of all possible ITS sequences, 10 clones per accession were randomly selected to sequence in a single direction by the TSINGKE Company (Beijing, China). The sequences were aligned 
online against the National Center for Biotechnology Information database to confirm the accuracy.

Multiple sequence alignments were generated using ClustalX software and manually adjusted using MEGA 5.0 software (Thompson et al. 1999, Tamura et al. 2011). In the initial phylogenetic analysis, only one sequence was retained when multiple sequences from the same accession formed a monophyletic group. Basic sequence data, including nucleotide frequencies, the transition/ transversion ratio, and variability, were recorded with $M E G A$ software. DnaSP 4.0 was employed to calculate the number of polymorphic sites, total number of mutations, number of haplotypes, haplotype diversity, and nucleotide diversity (Rozas et al. 2003).

Three sequence matrices (I, II, and III) were used to examine phylogenetic relationships, and putative relative species were included in each data set. Matrix I comprised all ITS sequences for tetraploid and relative species in Triticeae, and was subjected to phylogenetic network analysis with SplitsTree 4 using the NeighborNet algorithm (Bryant and Moulton 2004, Huson and Bryant 2005). To clarify the relationship between tetraploid wheat and Aegilops, matrix II, including one copy sequence of tetraploid wheat in a monophyletic group, Aegilops, and the putative A genome donor, was analyzed with the neighborjoining (NJ) algorithm using MEGA 5.0. A bootstrap analysis with 1000 replicates was performed to statistical support for the NJ tree topology. To further explore the evolution of ITS sequences in tetraploid wheat, matrix III, which comprised the ITS sequences for all accessions of tetraploid wheat, was subjected to a median-joining (MJ) network analysis using Network v5.0 software (Allaby and Brown 2001).

Fluorescence in situ hybridization analysis: In this study, we used pSc119.2 and pTa535 as probes. The probe pSc119.2 (6-FAM-5'CCGTTTTGTGGACTA TTACTCACCGCTTTGGGGTCCCATAGCTAT3') from rye repetitive sequences was used to determine the B genome chromosomes of wheat. The probe pTa535 (Tamra-5'AAAAACTTGACGCACGTCACGTACA A A T T G G A C A A A C T C T T T C G G A G T A T C A GGGTTTC3') from wheat repetitive sequences hybridizes preferentially to A and D genome chromosomes (Tang et al. 2014). Thus, binding of the two probes can be used to identify the wheat A and B chromosomes. Both probes were synthesized by Invitrogen (Shanghai, China). The FISH procedure followed the method of Han et al. (2006), with slight modifications. The probe mixture $\left(0.35 \mathrm{~mm}^{3}\right.$ of each probe in $2 \times \mathrm{SSC}$ and $1 \times$ TRIS-EDTA buffer, $\mathrm{pH} 7.0$, total volume $10 \mathrm{~mm}^{3}$ ) was dropped on a slide, covered with a coverslip, stored in a moist box at $37^{\circ} \mathrm{C}$ for $2 \mathrm{~h}$ and washed in $2 \times \mathrm{SSC}$ at room temperature. Chromosomes were counterstained with DAPI (4-6-diamino-2phenylindole) solution (Vector Laboratories, Burlingame, CA, USA). Photomicrographs of FISH chromosomes were taken with an Olympus BX-51 (Tokyo, Japan) microscope equipped with a $D P-70 C C D$ camera and all images were processed with Photoshop CS 5.0 (Adobe Systems, San Jose, CA, USA). Excel software was used to count the number of signal sites, and SLT NTSys2.1e software was used to normalize the matrix of signal sites, calculating coefficient of genetic similarity and neighbor-joining (NJ) algorithm to create a cluster analysis (Mantel 1967).

Meiotic pairing analysis: The $F_{1}$ hybrids included in this study are listed in Table 1. Stages of meiosis were determined in acetocarmine squashes of one of three anthers per flower. At the appropriate stage, the remaining two anthers were fixed in a mixture of absolute ethanol, chloroform, and acetic acid $(6: 3: 1, \mathrm{v} / \mathrm{v} / \mathrm{v}) \mathrm{kept}$ in a refrigerator for $24 \mathrm{~h}$, then stored at $4{ }^{\circ} \mathrm{C}$ in $70 \%(\mathrm{v} / \mathrm{v})$ alcohol. At least 30 pollen mother cells (PMCs) per plant were identified.

\section{Results}

A total of 70 ITS sequences for tetraploid wheat accessions were obtained by PCR amplification, cloning, and sequencing. For T. turgidum ssp. carthlicum accession PI532494 ITS copies were obtained from the A genome only, whereas for the remainder of the tetraploid wheat accessions ITS sequences were obtained from the B or $\mathrm{G}$ genomes. The amplified ITS sequence consisted of the ITS1 region, 5.8S rRNA gene, and ITS2 region. The total length of the sequence was $594-608 \mathrm{bp}$, of which the ITS1 region was 216 - $218 \mathrm{bp}$, the ITS2 region was 214 - $216 \mathrm{bp}$, and the 5.8S gene was $164-165 \mathrm{bp}$. The number of loci was 606, including 330 conserved characters, 268 variable characters, and 146 parsimony informative characters. The four residue frequencies were $\mathrm{A}=21.5 \%, \mathrm{~T} / \mathrm{U}=17.3 \%$, $\mathrm{G}=28.3 \%$, and $\mathrm{C}=32.9 \%$, respectively. The transition/ transversion ratio was 2.66. In the multiple sequence alignment generated with Clustal $W$, no indel was detected among the tetraploid wheat ITS sequences. The number of haplotypes (h) among the ITS sequences was 101, and the haplotype (gene) diversity (Hd) was 0.989 . The nucleotide diversity (Pi) value of 0.03672 indicated that the tetraploid wheat ITS sequences showed high genetic diversity.

To explore the taxonomic status of tetraploid wheat in the Triticeae, a network analysis including relative species was performed using SplitsTree 4.0. The network showed that the ITS sequences were split into two major clades (Fig. 1 Suppl.). Clade I originated from non-B and non-G genome types, which included all of the relative species with the exception of Aegilops species. Tetraploid wheat, as expected, was clustered with Aegilops species, and hexaploid wheat was included in Clade II. The Clade II comprised two subclades, which consisted of sequences from the B and G genomes. Subclade I consisted of the sequences from T. timopheevii and Aegilops species. In Subclade II, one, two, or three copies of ITS sequences were obtained from the accessions of T. turgidum.

To further examine the phylogenetic relationships between tetraploid wheat and the diploid donor species, all of the tetraploid wheat ITS sequences were included in a NJ analysis, together with five putative donors of Aegilops in the Triticeae (Fig. 1). Only one sequence was retained in the data set when the same accession was clustered in 


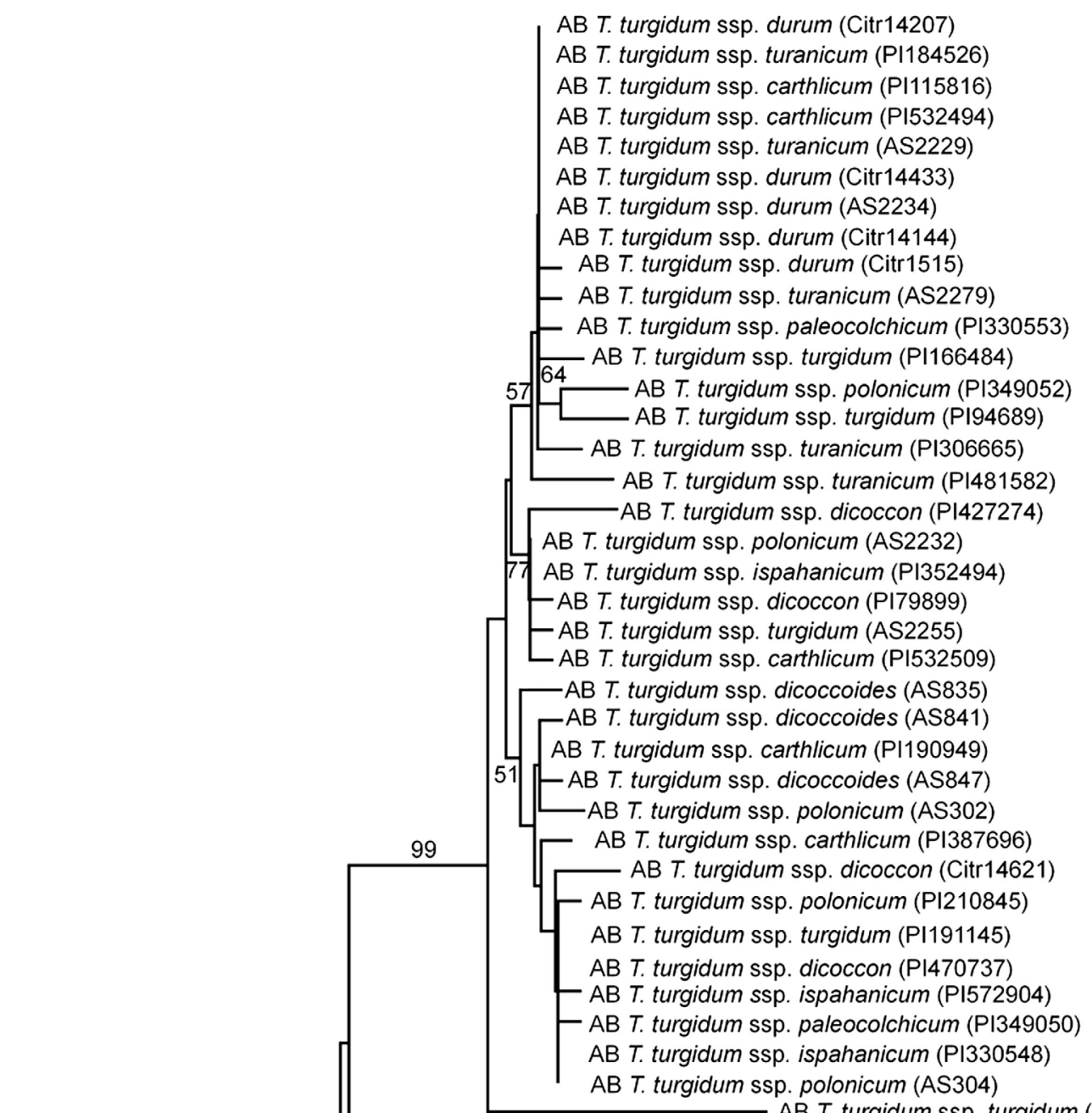

AB T. turgidum ssp. durum (Citr14207)

AB T. turgidum ssp. turanicum (PI184526)

turgidum ssp. carthlicum (PI115816)

AB $T$ turgidum SSp. turanicum (AS2229)

AB T. turgidum ssp. durum (Citr14433)

Turgidum ssp. durum (AS2234)

AB T. turgidum ssp. turanicum (AS2279)

AB T. turgidum ssp. paleocolchicum (PI330553)

64 AB T. turgidum ssp. turgidum (PI166484)

AB T. turgidum ssp. polonicum (PI349052)

AB T. turgidum ssp. turanicum (PI481582)

AB T. turgidum ssp. dicoccon (PI427274)

AB T. turgidum ssp. polonicum (AS2232)

AB T turgidum ssp. turgidum (AS2255)

AB T. turgidum ssp. carthlicum (PI532509)

turgidum Ssp. dicoccoides (AS835

AB T. turgidum ssp polonicum (PI210845)

AB T. turgidum ssp. turgidum (PI191145)

AB T. turgidum ssp. dicoccon (PI470737)

AB T. turgidum ssp. paleocolchicum (PI349050)

AB T. turgidum ssp. polonicum (AS304)

89 AG T. timopheevii ssp. araraticum (AS272)

AB T turgidum ssp. turgidum (AS2233)

58

56 - AG T. timopheevii ssp. araraticum (PI427302)

AG T. timopheevii ssp. araraticum (Pl427365)

AG T. timopheevii ssp. araraticum (PI503298)

AG T. timopheevii ssp. araraticum (PI427366)

93 AG T. timopheevii ssp. timopheevii (PI341804)

75-AG T. timopheevii ssp. timopheevii (AS274)

72 AG T. timopheevii ssp. timopheevii (PI94761)

$S$ Ae. sharonensis

93 S Ae. speltoides

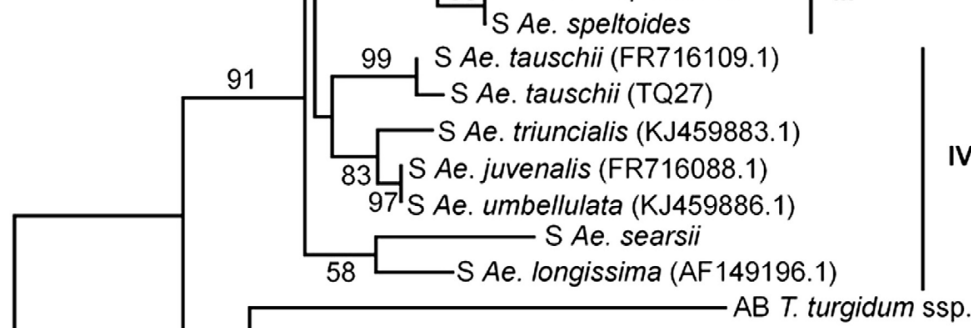

$84 \underset{62}{62}$ A T. monococcum

IV

0.01

Fig. 1. A neighbor-joining (NJ) tree derived from internal transcribed spacer region (ITS) sequences of tetraploid wheat and putative diploid donor species. The NJ tree divides all the ITS sequences into five clades. The species name and accession number are indicated for each taxon. Bootstrap support values greater than $50 \%$ are shown above the branches. 
a monophyletic group. Five major clades were resolved. In Clade I, all T. turgidum sequences were grouped with high bootstrap support (99\%), representing the B genome sequences. Clade I have three subclades with the varieties of T. turgidum separately, Clade II included all T. timopheevii sequences with the G genome sequences, Clade III consisted of two Ae. speltoides accessions and one Ae. sharonensis accession. The remainder of the Aegilops species formed Clade IV. Triticum turgidum ssp. carthlicum PI532494 was grouped with T. monococcum and T. urartu in Clade V. To highlight the relationships among haplotypes of the ITS sequence, a network analytical method was used. A MJ analysis of the haplotype data showed the relationship between T. timopheevii and $T$. turgidum (Fig. 2), which corresponded to clades revealed in the NJ phylogeny. All haplotypes derived from T. turgidum or T. timopheevii were clustered together, respectively. The MJ analysis further divided T. turgidum into major four parts. All T. turgidum ssp. turanicum and T. turgidum ssp. durum sequences were included in subgroup I. Triticum turgidum ssp. dicoccon (PI79899 and PI427274), T. turgidum ssp. polonicum (AS2232), T. turgidum ssp. ispahanicum (PI 352494), T. turgidum ssp. turgidum (AS2255), and T. turgidum ssp. carthlicum (PI532509) formed subgroup II. Most accessions of T. turgidum ssp. dicoccoides were included in subgroup III. Subgroup IV consisted of five haplotypes, including T. turgidum ssp. dicoccon (PI470737 and Citr14621), T. turgidum ssp. polonicum (AS304 and PI210845), T. turgidum ssp. turgidum (PI191145), T. turgidum ssp. paleocolchicum (PI349050), and T. turgidum ssp. ispahanicum (PI572904).

To further explore phylogenetic relationships and genetic diversity of tetraploid wheat, chromosomal differentiation in tetraploid wheat accessions was analyzed by FISH using probes that targeted chromosomal sites mainly in the A, B, and $\mathrm{G}$ genomes.

On the A genome, the probes pSc119.2 and pTa535 produced abundant signals, and many differences in signals were observed on chromosomes 1A, 2A, 4A, and 5A among T. turgidum and T. timopheevii accessions (Fig. 3). The pSc119.2 signals were only observed on chromosome $1 \mathrm{AS}, 1 \mathrm{AL}, 2 \mathrm{AS}, 2 \mathrm{AL}, 4 \mathrm{AL}, 5 \mathrm{AS}$, and $5 \mathrm{AL}(\mathrm{S}$ and $\mathrm{L}$ represent the short and long arm of the chromosome, respectively), and hybridization patterns were variable. The terminal pSc119.2 signals on chromosome $1 \mathrm{AS}$ was only detected in five accessions, including T. turgidum ssp. dicoccoides (AS841 and PI470947), T. turgidum ssp. ispahanicum (PI330548), T. turgidum ssp. paleocolchicum (PI349050), and T. turgidum ssp. carthlicum (PI387696) accessions. Two accessions of $T$. timopheevii ssp. timopheevii were identified by strong subterminal signals of $\mathrm{pSc} 119.2$ on chromosome 1AL. This probe hybridized to the terminal region of chromosome $2 \mathrm{AS}$ only in T. turgidum ssp. turgidum (AS2255). The strong terminal

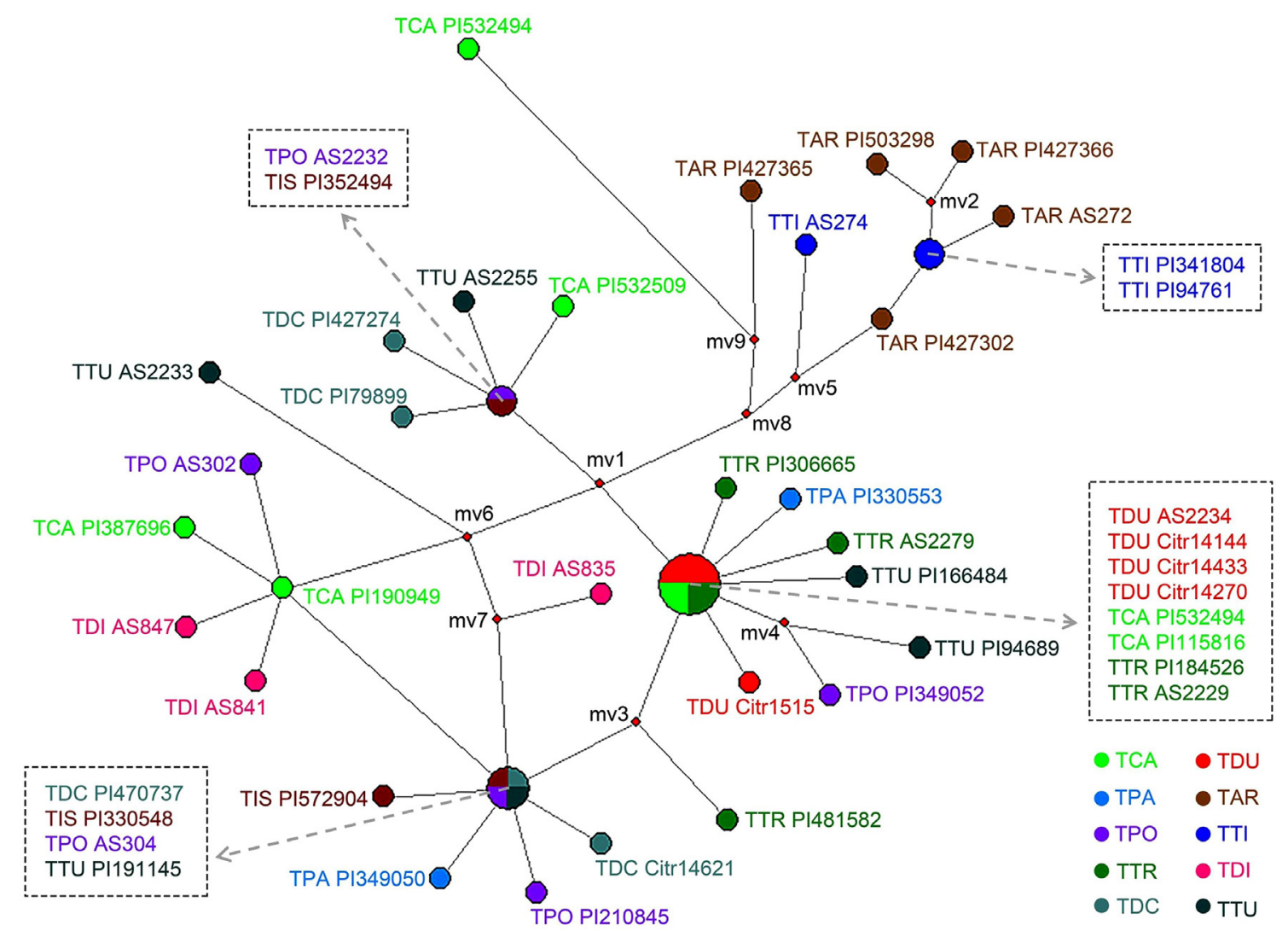

Fig. 2. A median-joining network derived from internal transcribed spacer region sequences of tetraploid wheat. Abbreviations for the species are listed in Table 1 Suppl. Each circle represents a haplotype, each color represents one species, and the node size is proportional to the number of haplotypes. Median vectors ( $\mathrm{mv}$ ) represent inferred unsampled nodes. Numbers on branches indicate a mutation site. 


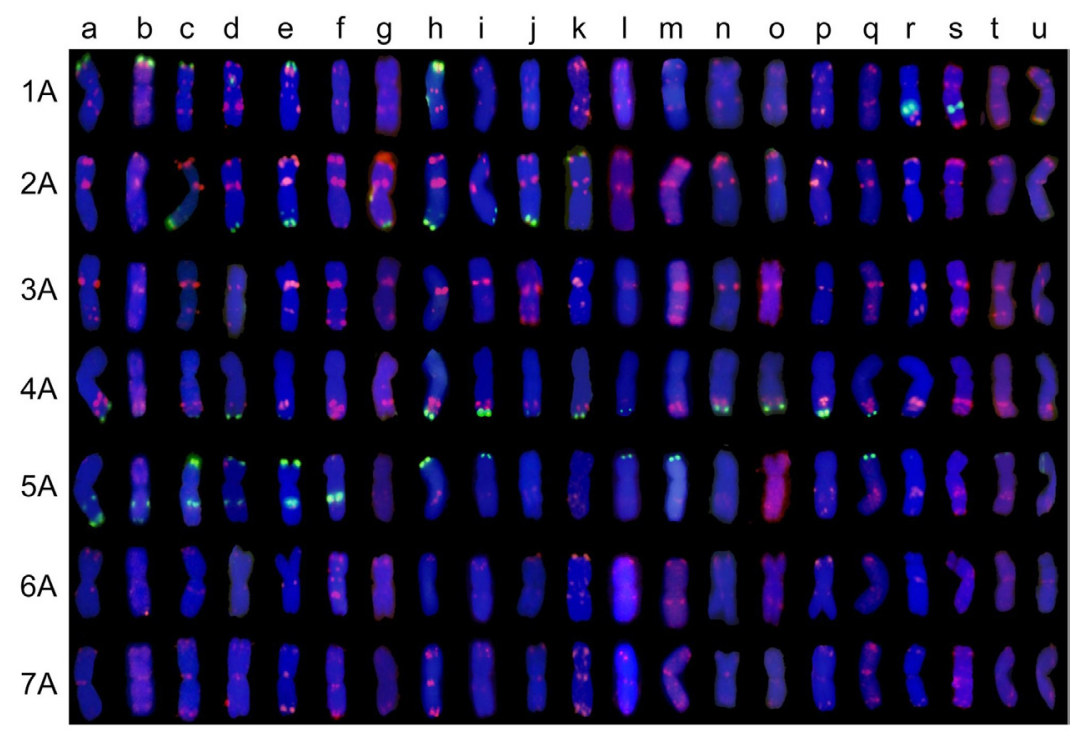

Fig. 3. Fluorescence in situ hybridization probe signals on genome chromosomes A of tetraploid wheat for pSc119.2 (green) and pTa535 (red). Columns a to u - Triticum turgidum ssp. dicoccoides (PI470947), T. turgidum ssp. dicoccoides (AS841), T. turgidum ssp. ispahanicum (PI330548), T. turgidum ssp. ispahanicum (PI572904), T. turgidum ssp. paleocolchicum (PI349050), T. turgidum ssp. dicoccon (Citr3686), T. turgidum ssp. dicoccon (PI427274), T. turgidum ssp. carthlicum (PI387696), T. turgidum ssp. carthlicum (PI115816), T. turgidum ssp. turgidum (PI191145), T. turgidum ssp. turgidum (AS2255), T. turgidum ssp. polonicum (PI366117), T. turgidum ssp. polonicum (AS304), T. turgidum ssp. turanicum (PI481582), T. turgidum ssp. turanicum (AS2229), T. turgidum ssp. durum (Citr14144), T. turgidum ssp. durum (Citr14433), T. timopheevii ssp. timopheevii (PI282933), T. timopheevii ssp. timopheevii (Citr15205), T. timopheevii ssp. araraticum (PI427366), and T. timopheevii ssp. araraticum (AS272).

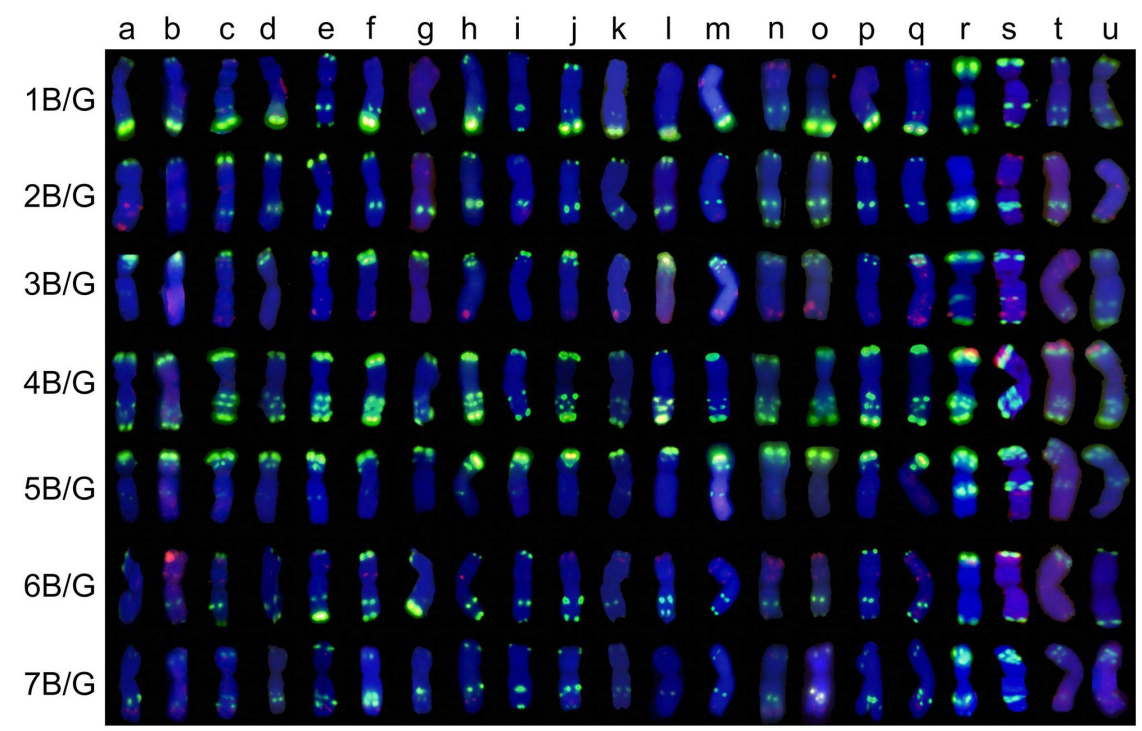

Fig. 4. Fluorescence in situ hybridization probe signals on genome chromosomes B and D of tetraploid wheat for pSc119.2 (green) and pTa535 (red). The accession numbers are the same as those listed in Fig. 3.

pSc119.2 signals on chromosome 2AL were found among seven accessions. The signal located in the terminal region of 4AL was observed in almost all accessions, excluding T. turgidum ssp. dicoccon (Citr3686), T. turgidum ssp. polonicum (AS304), T. timopheevii ssp. timopheevii, and T. timopheevii ssp. araraticum. The hybridization patterns on chromosome 5AL of $T$. turgidum ssp. dicoccoides (AS841 and PI470947) differed from other accessions with clear terminal and subterminal signals. T. turgidum ssp. ispahanicum (PI330548 and PI572904) also showed strong signals on the terminal region of chromosome 5AS and subterminal region of $5 \mathrm{AL}$, respectively. Signal sites for the pTa535 probe were completely present on $1 \mathrm{~A}-7 \mathrm{~A}$ chromosomes of all the accessions. We found that the pTa535 site diversities on chromosome 1A, 2A, and 7A were low. In the terminal region of chromosome $3 \mathrm{AL}$, only T. turgidum ssp. paleocolchicum (PI349050), T. turgidum ssp. carthlicum (PI387696), T. turgidum ssp. carthlicum (PI115816), T. turgidum ssp. polonicum (PI366117), and T. turgidum ssp. turanicum (PI481582) lacked red pTa535 


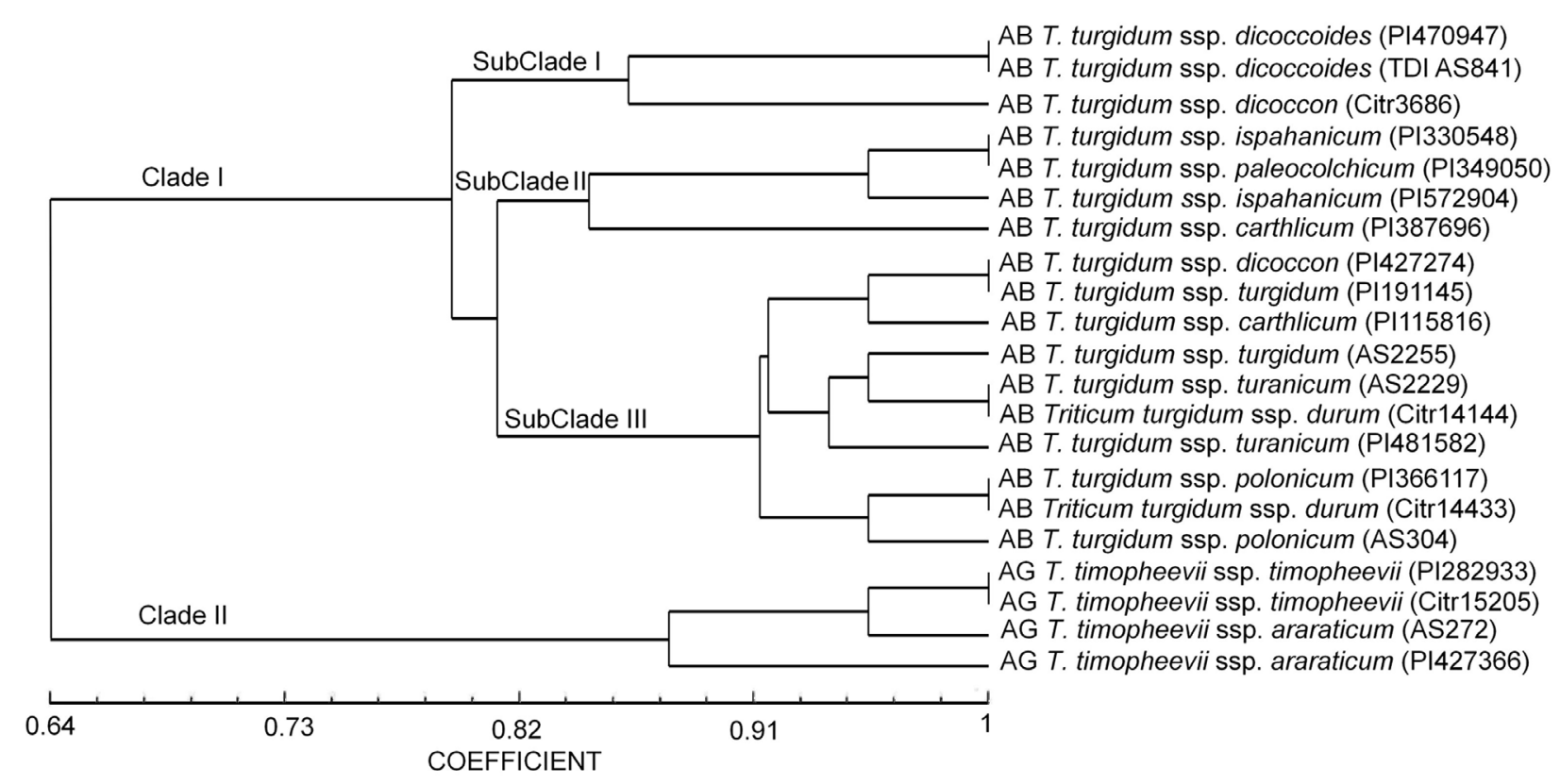

Fig. 5. A neighbor-joining tree derived from fluorescence in situ hybridization signal sites in tetraploid wheat.

signals. The probe pTa535 gave strong signals on the terminal and the terminal-subterminal regions on the long arm of chromosome $4 \mathrm{~A}$, respectively. The signal sites of pTa535 on chromosome 5AL was present in all accessions except for T. turgidum ssp. dicoccoides (PI470947), T. turgidum ssp. dicoccoides (AS841), T. turgidum ssp. ispahanicum (PI330548), T. turgidum ssp. ispahanicum (PI572904), T. turgidum ssp. paleocolchicum (PI349050), and T. turgidum ssp. dicoccon (Citr3686). Furthermore, only $T$. turgidum ssp. carthlicum (PI387696) lacked the faint signals near the centromere on chromosome 6AL.

Signal sites for the probe pSc119.2 were observed on all B-genome chromosomes of all accessions (Fig. 4). Hybridization differences for this probe were observed on all chromosomes except $3 \mathrm{~B}$ and $4 \mathrm{~B}$. Three pairs of signals were detected on chromosome $1 \mathrm{~B}$ for all accessions except T. turgidum ssp. ispahanicum (PI330548), T. turgidum ssp. dicoccon (Citr3686 and PI427274), T. turgidum ssp. polonicum (AS304 and PI366117), T. turgidum ssp. turanicum (PI481582 and AS2229), and T. turgidum ssp. durum (AS2234). The most characteristic signal patterns were generated in two accessions T. turgidum ssp. turanicum with two pairs of signals on chromosome $2 \mathrm{BL}$ and one pair of signals on chromosome $2 \mathrm{BS}$. However, only terminal pTa119.2 signals were detected on chromosome $2 \mathrm{BS}$ of the accessions T. turgidum ssp. dicoccoides. On chromosome $6 \mathrm{~B}$, different signal combinations were observed among 11 taxa. Three pairs of signals on chromosome 7B were only observed in T. turgidum ssp. turgidum (PI191145) and T. turgidum ssp. carthlicum (PI115816). Signal sites for pTa535 were mainly apparent in the terminal region of chromosomes 3BL and 6BS, and showed less diversity. Chromosome 2BL of T. turgidum ssp. dicoccoides carried terminal and subterminal pTa535 signals, which differed from other accessions.

We found that all G-genome chromosomes could be unambiguously identified using a combination of pSc119.2 and pTa535 repeats, and the G-genome chromosomes of T. timopheevii and B-genome chromosomes of T. turgidum exhibited significant variation on all seven homologous chromosomes in the probe hybridization patterns (Fig. 4). Chromosome $1 \mathrm{G}$ carried strong terminal pSc119.2 signals on both arms, and strong pSc119.2 signals were found on the subterminal region of the long arm. Chromosome $2 \mathrm{G}$ of $T$. timopheevii ssp. timopheevii was characterized by strong subterminal signals of pSc119.2 on long arm and faint terminal pTa535 signals on the short arm, which differed from those of $T$. timopheevii ssp. araraticum. Clear terminal pSc119.2 signals, subterminal pTa535 signals, and faint pSc119.2 signals near the centromere were detected on the long arm of chromosome $3 \mathrm{G}$, and the short arm produced strong telomeric pSc119.2 signals. Strong terminal and two subterminal pSc119.2 signals were detected on the long arm of chromosome $4 \mathrm{G}$, and interestingly, the short arm simultaneously produced strong telomeric pSc119.2 and pTa535 signals. Compared to B-genome chromosomes, chromosome $5 \mathrm{G}$ had strong pSc119.2 signals near the centromere region, chromosome 6G lacked specific pSc119.2 signals subterminally on the long arm, and chromosome $7 \mathrm{G}$ showed strong pSc119.2 signals on the subterminal region of the short arm.

Given that copies of ITS sequences for tetraploid wheat were only obtained from the B or G genomes, cluster analysis of FISH signal site statistics located in the A genome was performed using the NJ method (Fig. 5). As expected, the 17 accessions of T. turgidum and 4 accessions of $T$. timopheevii clustered on distinct branches, and the cladogram showed a similarly topology 
Table 1. Chromosome pairing at meiotic metaphase I in pollen mother cells of $F_{1}$ hybrids.

\begin{tabular}{|c|c|c|c|c|c|c|}
\hline \multirow[t]{2}{*}{ Hybrid } & \multicolumn{2}{|c|}{ Chromosome pairing } & \multirow[b]{2}{*}{ II(ring) } & \multirow[b]{2}{*}{ II(rod) } & \multirow[b]{2}{*}{ III } & \multirow[b]{2}{*}{ IV } \\
\hline & I & II(total) & & & & \\
\hline $\begin{array}{l}\text { T. timopheevii ssp. araraticum PI } 427366 \\
\times \text { T. turgidum ssp. polonicum PI } 210845\end{array}$ & $8.38(4-15)$ & $9.63(5-12)$ & $3.36(1-5)$ & $6.27(4-8)$ & $0.12(0-1)$ & 0 \\
\hline $\begin{array}{l}\text { T. turgidum ssp. turanicum AS2229 } \\
\times \text { T. turgidum ssp. polonicum PI } 366117\end{array}$ & $0.20(0-2)$ & $13.90(13-14)$ & $1.85(0-6)$ & $12.05(8-14)$ & 0 & 0 \\
\hline $\begin{array}{l}\text { T. turgidum ssp. durum Citr14144 } \\
\times \text { T. turgidum ssp. dicoccoides AS847 }\end{array}$ & $1.84(0-6)$ & $13.08(11-14)$ & $3.94(0-7)$ & $9.14(0-13)$ & 0 & 0 \\
\hline $\begin{array}{l}\text { T. turgidum ssp. dicoccoides AS841 } \\
\times \text { T.turgidum L. cv. Ailanmai }\end{array}$ & $1.48(0-6)$ & $13.26(11-14)$ & $6.17(0-12)$ & $7.09(2-10)$ & 0 & 0 \\
\hline $\begin{array}{l}\text { T. turgidum ssp. dicoccon PI } 427274 \\
\times \text { T. turgidum ssp. durum Citr } 14144\end{array}$ & $0.88(0-4)$ & $13.56(12-14)$ & $2.66(0-6)$ & $10.90(6-14)$ & 0 & 0 \\
\hline $\begin{array}{l}\text { T. turgidum ssp. dicoccon PI } 427274 \\
\times \text { T. turgidum ssp. turgidum AS2255 }\end{array}$ & $0.68(0-4)$ & $13.22(10-14)$ & $1.71(0-5)$ & $11.51(8-14)$ & 0 & $0.22(0-2)$ \\
\hline $\begin{array}{l}\text { T. turgidum ssp. turanicum PI } 481582 \\
\times \text { T. turgidum ssp. dicoccon PI } 427274\end{array}$ & $0.68(0-4)$ & $13.66(12-14)$ & $2.93(0-7)$ & $10.73(6-14)$ & 0 & 0 \\
\hline $\begin{array}{l}\text { T. turgidum ssp. dicoccon PI } 427274 \\
\times \text { T. turgidum ssp. carthlicum PI } 387696\end{array}$ & $0.54(0-2)$ & $13.73(13-14)$ & $1.86(0-5)$ & $11.87(9-14)$ & 0 & 0 \\
\hline $\begin{array}{l}\text { T. turgidum ssp. turanicum AS2229 } \\
\times \text { T. turgidum ssp. durum Citr14144 }\end{array}$ & $0.46(0-4)$ & $13.77(12-14)$ & $2.76(0-4)$ & $11.01(10-14)$ & 0 & 0 \\
\hline $\begin{array}{l}\text { T. turgidum ssp. turgidum AS2255 } \\
\times \text { T. turgidum ssp. polonicum PI } 210845\end{array}$ & $0.20(0-4)$ & $13.44(12-14)$ & $1.52(0-5)$ & $11.92(10-14)$ & 0 & $0.23(0-1)$ \\
\hline $\begin{array}{l}\text { T. turgidum ssp. dicoccon PI } 427274 \times \\
\text { T. turgidum ssp. dicoccoides AS } 841\end{array}$ & $0.20(0-2)$ & $13.85(13-14)$ & $2.38(0-5)$ & $11.47(9-14)$ & 0 & 0 \\
\hline $\begin{array}{l}\text { T. turgidum ssp. carthlicum PI38769 } \\
\times \text { T. turgidum ssp. durum Citr14144 }\end{array}$ & $0.18(0-2)$ & $13.91(13-14)$ & $1.78(0-5)$ & $12.13(9-14)$ & 0 & 0 \\
\hline $\begin{array}{l}\text { T. turgidum ssp. turgidum PI166484 } \\
\times \text { T. turgidum ssp. carthlicum PI115816 }\end{array}$ & $0.12(0-2)$ & $13.48(12-14)$ & $1.53(0-6)$ & $11.95(9-14)$ & 0 & $0.23(0-1)$ \\
\hline $\begin{array}{l}\text { T. turgidum ssp. carthlicum PI } 387696 \\
\times \text { T. turgidum ssp. Turgidum AS2255 }\end{array}$ & $0.08(0-2)$ & $13.56(12-14)$ & $1.46(0-6)$ & $12.10(9-14)$ & 0 & $0.20(0-1)$ \\
\hline
\end{tabular}

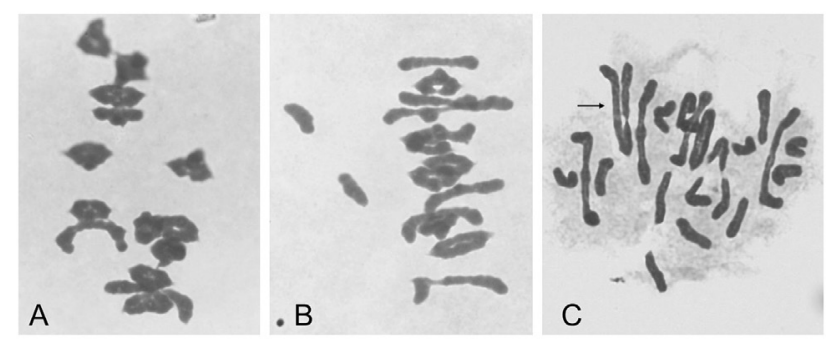

Fig. 6. Meiotic pairing behavior in $\mathrm{F}_{1}$ hybrids. A - Triticum turgidum ssp. dicoccon (PI427274) $\times$ T. turgidum ssp. durum (CItr14144), $2 \mathrm{n}=28=12 \mathrm{II}$ (Ring) $+2 \mathrm{II}$ (Rod); $B-T$. turgidum ssp. durum (CItr14144 $\times$ T. turgidum ssp. dicoccoides (AS847), $2 \mathrm{n}=28=2 \mathrm{I}+6 \mathrm{II}$ $($ Ring $)+7$ II (Rod); $C$ - T. timopheevii ssp. araraticum $(\mathrm{PI} 427366) \times$ T. turgidum ssp. polonicum $(\mathrm{PI} 210845), 2 \mathrm{n}=28=15 \mathrm{I}+1 \mathrm{II}(\mathrm{Ring})$ +4 II (Rod) +1 IIII. The arrow indicates the trivalent.

to that of the NJ phylogenetic tree constructed with MEGA. These results indicated that $T$. turgidum was divided into three subclades, which represented potential evolutionary lineages. Importantly, two T. turgidum ssp. dicoccoides
(AS841 and PI470947) and one T. turgidum ssp. dicoccon (Citr3686) were placed in subclade I. Two T. turgidum ssp. ispahanicum (PI330548 and PI572904), one T. turgidum ssp. paleocolchicum (PI349050), and one T. turgidum 
ssp. carthlicum (PI387696) were clustered in subclade II. The remainder accessions of T. turgidum were assigned to subclade III, including all T. turgidum ssp. turanicum, T. turgidum ssp. durum, one T. turgidum ssp. turgidum (AS2255), and one T. turgidum ssp. polonicum (AS304).

The above results did not directly indicate the classification systems of tetraploid wheat. Chromosome pairing in $F_{1}$ hybrids of tetraploid wheat was examined to detect genomic affinities (Table 1, Fig. 6). All hybrids derived from $T$. turgidum were of genomic composition $\mathrm{AABB}$, with $2 \mathrm{n}=4 \mathrm{x}=28$. The hybrids of $T$. turgidum ssp. dicoccoides showed a high number of univalents in metaphase I cells. The $\mathrm{F}_{1}$ hybrid of T. turgidum ssp. dicoccoides $\times T$. turgidum L. cv. Ailanmai showed 0 to 6 univalents, with an average of 1.48. The average pairing was 1.84 univalents, 13.08 bivalents in the $\mathrm{F}_{1}$ hybrid of T. turgidum ssp. durum $\times$ T. turgidum ssp. dicoccoides. When $T$. turgidum ssp. turgidum was a parent, a low frequency of tetravalents was found. These results indicated that hybrids from naked tetraploid wheat showed a high frequency of bivalents and a low frequency of univalents. In the $\mathrm{F}_{1}$ hybrid of $T$. timopheevii ssp. araraticum $(\mathrm{PI} 427366) \times T$. turgidum $\mathrm{ssp}$. polonicum (PI210845), meiotic pairing was characterized by a high frequency of univalents ranging from 4 to 15 , with an average of 8.38 per cell.

\section{Discussion}

Triticum turgidum possesses excellent agronomic traits and is useful as breeding material to transfer target traits to $T$. aestivum. However, the uncertain evolutionary history and classification of tetraploid wheat is not conducive to effective utilization of tetraploid wheat resources. Exploration of the phylogenetic relationships of tetraploid wheat is beneficial to protect the genetic diversity of tetraploid wheat and broaden the genetic basis of T. aestivum.

The taxonomy of tetraploid wheat is controversial. Triticum has been recognized as a distinct genus in the Triticeae since Linnaeus's time (Goncharov et al. 2011). As Aegilops and Triticum are closely related, many taxonomists propose that the two genera should be combined (Yamane and Kawahara 2005). With a clear understanding of the phylogenetic relationship between Aegilops and Triticum, the B and G genome of Triticum were thought to be derived from Aegilops (Huang et al. 2002). MacKey $(1966,2005)$ defined Triticum to include two intergeneric hybrids of $\times$ Triticosecale Wittm, and given that some lines of Triticum cross more readily with Secale than with Aegilops, and that immunochemistry shows Triticum and Secale to be more similar than Triticum and Aegilops, thus proposed that Triticum and Secale should be merged. In the present study, based on network analysis of ITS sequences using SplitsTree, all relative species in the Triticeae with the exception of Aegilops species showed a common topology, whereas tetraploid wheat, Aegilops species, and hexaploid wheat were clustered in Clade II. These results showed that Triticum and Secale were not closely related, thus supporting Bowden's classification (1959) and opposing the treatment of $\times$ Triticosecale by MacKey (1966). To further illustrate the relationship between tetraploid wheat and Aegilops species, the NJ phylogenetic analysis indicated that tetraploid wheat and Aegilops clustered separately on different branches, and Ae. speltoides and one accession of Ae. sharonensis were more closely related to tetraploid wheat than to other Aegilops species. This finding indicated that Aegilops plus Triticum was not monophyletic, thus rejecting their treatment as a single genus (Bowden 1959).

On the basis of glume morphology, Linnaeus treated tetraploid wheat as T. polonicum (Goncharov 2011). With regard to tetraploid wheat, Bowden (1959) recognized one species, T. turgidum, three varieties, two variants, and eight cultivars. Given cytogenetic evidence and geographic distribution, Morris and Sears (1967) considered tetraploid wheat to consist of two species. Provan et al. (2004) observed that the cytoplasmic types were very similar, supporting the hypothesis for the monophyletic origin of tetraploid wheat. However, Kilian et al. (2007) detected a large number of differences between the B and $\mathrm{G}$ genomes based on different gene loci, supporting the hypothesis for two independent origins of tetraploid wheat. In the present network analysis and phylogenetic analysis, T. turgidum and T. timopheevii were placed on different branches, indicating that $T$. turgidum was distantly related to T. timopheevii. The FISH signal sites of T. turgidum and T. timopheevii are very different, and the signal distribution on each chromosome has been mutated. This variation was far greater than the degree of variation among accessions of T. turgidum, indicating that T. turgidum and T. timopheevii show very high genetic differentiation. The $\mathrm{F}_{1}$ hybrid of T. timopheevii ssp. araraticum PI427366 $\times$ T. turgidum ssp. polonicum PI210845 showed a high frequency of univalent at metaphase I, which is an indication of genomic incompatibility. These findings indicate that T. turgidum and $T$. timopheevii are two distantly related species with different origins.

Numerous classifications of wheats based on different criteria were used by researchers, which has caused ongoing confusion and generic and species concepts remain controversial. Among the two most widely used classifications of tetraploid wheat, MacKey (1966) considered all tetraploid wheat to be subspecies. In contrast, Dorofeev's classification treats all forms of tetraploid wheat as species (Dorofeev et al. 1979). The present study of meiotic behavior of $F_{1}$ hybrids among tetraploid wheat accessions in metaphase I cells indicated that the frequency of univalents was low (means $<2$ ), and the NJ phylogenetic analysis was consistent with meiotic behavior, which indicated that all forms of T. turgidum should not be considered species, thus supporting MacKey's classification system.

Archaeological and genetic evidence suggests that domesticated T. turgidum originated from T. turgidum ssp. dicoccoides in the Fertile Crescent, and that naked tetraploid wheat originated from domesticated T. turgidum ssp. dicoccon (Zohary et al. 2000, Salamini et al. 2002, Matsuoka 2011). Tang et al. (2017) indicated that 
T. turgidum ssp. dicoccon and T. turgidum ssp. dicoccoides grouped a subclade based on the nuclear DMC1 gene, and concluded that at least two intermediary subspecies were involved in the evolution of T. turgidum. The $\mathrm{F}_{1}$ hybrids of T. turgidum ssp. dicoccoides showed a higher frequency of univalents than other hybrids. In the cluster analysis of FISH results, two T. turgidum ssp. dicoccoides (TDI AS841 and TDI PI470947) and one T. turgidum ssp. dicoccon (TDC Citr3686) were clustered in subclade I. These results show the special status of T. turgidum ssp. dicoccoides in T. turgidum. Triticum turgidum ssp. dicoccoides contains two populations, one from the west, including Jordan, Israel, Lebanon, and Syria, and the other from the Middle East, including Iran, Iraq, and Turkey. Many studies have shown that T. turgidum ssp. dicoccoides in the Middle East is the ancestral species of domesticated tetraploid wheat (Özkan et al. 2005, 2011, Matsuoka et al. 2011, Oliveira et al. 2012). In the present study, accessions of T. turgidum ssp. dicoccoides from the western population clustered with domesticated tetraploid wheat, which is inconsistent with the results of Özkan et al. (2011) and Oliveira et al. (2012). With regard to meiotic behavior of $F_{1}$ hybrids, and the results of NJ phylogenetic analysis and network analysis, the forms of T. turgidum were not distinguished except for $T$. turgidum ssp. dicoccoides. Therefore, we advocate continued recognition of $T$. turgidum ssp. dicoccoides as a subspecies and the treatment of other forms of T. turgidum as varieties.

\section{References}

Allaby, R.G., Brown, T.A.: Network analysis provides insights into evolution of 5S rDNA arrays in Triticum and Aegilops. Genetics 157: 1331-1341, 2001.

Bowden, W.N.: The taxonomy and nomoenclature of the wheats, barleys and ryes and their wild relatives. - Can. J. Bot. 37: 637-684, 1959.

Bryant, D., Moulton, V.: Neighbor-Net: an agglomerative method for the construction of phylogenetic networks. - Mol. Biol. Evol. 21: 255-265, 2004.

Chen, X.M., Luo, Y.H., Xia, X.C., Xia, L.Q., Chen, X., Ren Z.L., Jia, J.Z.: Chromosomal location of powdery mildew resistance gene $P m 16$ in wheat using SSR marker analysis. Plant Breed. 124: 225-228, 2005.

Dorofeev, V.F., Filatenko, A.A., Migushova, E.F., Udaczin, R.A., Jakubziner, M.M.: Pshenitsa [Wheat]. - In: Dorofeev, V.F., Korovin, O.N. (ed.): Cultivated Flora of the USSR. Pp. 364. Kolos Publ., Leningrad 1979. [In Russ.]

Doyle, J.J., Doyle, J.L.: Isolation of plant DNA from fresh tissue. - Focus 12: 13-15, 1990.

Forte, P., Virili, M.E., Kuzmanović, L., Moscetti, I., Gennaro, A., D'Ovidio, R., Ceoloni, C.: A novel assembly of Thinopyrum ponticum genes into the durum wheat genome: pyramiding Fusarium head blight resistance onto recombinant lines previously engineered for other beneficial traits from the same alien species. - Mol. Breed. 34: 1701-1716, 2014.

Gerechmr-Amitai, Z.K., Van-Silthout, C.H., Grama, A., Kleitman, F.: Yr15 - a new gene for resistance to Puccinia striiformis in Triticum dicoccoides sel. G-25. - Euphytica 43: 187-190, 1989.

Goncharov, N.P., Golovnina, K.A., Kondratenko, E.Y., Komatsuda, T.: Taxonomy and molecular phylogeny of natural and artificial wheat species. - Breed. Sci. 59: 492-498, 2009.

Goncharov, N.P.: Genus Triticum L. taxonomy: the present and the future. - Plant Syst. Evol. 295: 1-11, 2011.

Han, F., Lamb, J., Birchler, J.: High frequency of centromere inactivation resulting in stable dicentric chromosomes of maize. - Proc. nat. Acad. Sci. USA 103: 3238-3243, 2006.

Hsiao, C., Chatterton, N.J., Asay, K.H., Jensen, K.B.: Phylogenetic relationships of the monogenomic species of the wheat tribe, Triticeae (Poaceae), inferred from nuclear rDNA (internal transcribed spacer) sequences. - Genome 38: 221223, 1995.

Huang, S.X., Sirikhachornkit, A., Su, X.J., Fairs, J., Gill B., Haselkorn R., Gornicki P.: Genes encoding plastid acetyl-CoA carboxylase and 3-phosphoglycerate kinase of the Triticum Aegilops complex and the evolutionary history of polyploid wheat. - Proc. nat. Acad. Sci. USA 99: 8133-8138, 2002.

Huson, D.H., Bryant, D.: Application of phylogenetic networks in evolutionary studies. - Mol. Biol. Evol. 23: 254-267, 2005.

Jauhar, P.P., Peterson, T.S.: Registration of DGE-1, a durum alien disomic addition line with resistance to Fusarium head blight. - J. Plant Reg. 2: 167-168, 2008.

Jauhar, P.P., Peterson, T.S., Xu, S.S.: Cytogenetic and molecular characterization of a durum alien disomic addition line with enhanced tolerance to Fusarium head blight. - Genome 52: 467-483, 2009.

Jauhar, P.P.: Durum wheat genetic stocks involving chromosome $1 \mathrm{E}$ of diploid wheatgrass: resistance to Fusarium head blight. - Nucleus 57: 19-23, 2014.

Kilian, B., Özkan, H., Deusch, O., Effgen, S., Brandolini, A., Kohl, J., Martin, W.: Independent wheat B and G genome origins in outcrossing Aegilops progenitor haplotypes. - Mol. Biol. Evol. 24: 217-227, 2007.

Kuzmanović, L., Gennaro, A., Benedettelli, S., Dodd, L.C., Quarrie, S.A., Ceoloni, C.: Structural functional dissection and characterization of yield-contributing traits originating from a group 7 chromosome of the wheatgrass species Thinopyrum ponticum after transfer into durum wheat. - J. exp. Bot. 65: 509-525, 2014.

Liu, Z.Y., Sun, Q.X., Xi, Z.F., Nevo, E., Yang, T. Molecular characterization of a novel powdery mildew resistance gene Pm30 in wheat originating from wild emmer. - Euphytica 123: 21-29, 2002.

MacKey, J.: Species relationship in Triticum. - In: MacKey, J. (ed.): Proceedings of the $2^{\text {nd }}$ International Wheat Genetics Symposium. Vol. 2. Pp. 237-275. Hereditas, Lund 1966.

MacKey, J.: The boundaries and subdivision of the genus Triticum. - In. MacKey, J. (ed.): Proceedings of the $12^{\text {th }}$ International Botanical Congress. Vol. 2. Pp. 1-23. Kolos Publ., Leningrad 1975.

MacKey, J.: Wheat: its concept, evolution and taxonomy. - In: Royo, C., Nachit, M.M., Fonzo, N.D. (ed.): Durum Wheat Breeding: Current Approaches and Future Strategies. Vol. 1. Pp. 35-94. CRC Press, Boca Raton 2005.

Mantel, N.: The detection of disease clustering and a generalized regression approach. - Cancer Res. 27: 209-220, 1967.

Matsuoka, Y.: Evolution of polyploid Triticum wheats under cultivation: the role of domestication, natural hybridization and allopolyploid speciation in their diversification. - Plant Cell Physiol. 52: 750-764, 2011.

Morris, R., Sears, E.R.: The cytogenetics of wheat and its relatives. - In: Ouissenberry, R. (ed.): Wheat and Wheat Improvement. Pp. 19-87. American Society of Agronomy, Madison 1967.

Muterko, A., Kalendar, R., Cockram, J., Balashova, I.: Discovery, evaluation and distribution of haplotypes and new alleles of 
the Photoperiod-Al gene in wheat. - Plant mol. Biol. 88: 149164, 2015 .

Oliveira, H.R., Campana, M.G., Jones, H., Hunt, H.V., Leigh, F., Redhouse, D.I.: Tetraploid wheat landraces in the Mediterranean basin: taxonomy, evolution and genetic diversity. - Plos ONE 7: e37063, 2012.

Özkan, H., Willcox, G., Graner, A., Salamini, F., Kilian, B.: Geographic distribution and domestication of wild emmer wheat (Triticum dicoccoides). - Genet. Resour. Crop Evol. 58: 11-53, 2011.

Özkan, H., Brandolini, A., Pozzi, C.S., Effgen, S., Wunder, J., Salamini, F.: A reconsideration of the domestication geography of tetraploid wheats. - Theor. appl. Genet. 110: 1052-1060, 2005.

Peng, J.H., Fahima, T., Röder, M.S., Li, Y.C., Dahan, A., Grama, A., Ronin, Y.I.: Microsatellite tagging of the striperust resistance gene $\mathrm{YrH} 52$ derived from wild emmer wheat, Triticum dicoccoides, and suggentive negative crossover interference on chromosome 1B. - Theor. appl. Genet. 98: 862-872, 1999.

Peleg, Z., Saranga, Y., Yaziei, A., Fahima, T., Ozturk, L.: Grain zinc, iron and protein concentrations and zinc-efficiency in wild emmer wheat under contrasting irrigation regimes. Plant Soil 306: 57-67, 2008.

Provan, J., Wolters, P., Caldwell, K.H., Powell, P.: High-resolution organellar genome analysis of Triticum and Aegilops sheds new light on cytoplasm evolution in wheat. - Theor. appl. Genet. 108: 1182-1190, 2004.

Reader, S.M., Miller, T.E.: The introduction into bread wheat of a major gene for resistance to powdery mildew from wild emmer wheat. - Euphytica 53: 57-60, 1991.

Rong, J.K., Millet, E., Manisterski, J., Feldman, M.: A new powdery mildew resistance gene: introgression from wild emmer into common wheat and RFLP-based mapping. Euphytica 115: 121-126, 2000.

Rozas, J., Sánchez del Barrio, J.C., Messeguer, X., Rozas, R.: DnaSP, DNA polymorphism analyses by the coalescent and the other methods. - Bioinformatics 19: 2496-2497, 2003.

Salamini, F., Özkan, H., Brandolini, A., Schäfer, P.R., Martin, W.: Genetics and geography of wild cereal domestication in the Near East. - Nat. Rev. Genet. 3: 429-441, 2002.

Sormacheva, I., Golovnina, K., Vavilova, V., Kosuge, K., Watanabe, N., Blinov, A., Goncharov N.P.: Q gene variability in wheat species with different spike morphology. - Genet. Resour. Crop Evol. 62: 837-852, 2015.

Takenaka, S., Mori, N., Kawahara, T.: Genetic variation in domesticated emmer wheat (Triticum turgidum L.) in and around Abyssinian Highlands. - Breed. Sci. 60: 212-227, 2010.

Takenaka, S., Kawahara, T.: Evolution and dispersal of emmer wheat (Triticum sp.) from novel haplotypes of Ppd-1 (photoperiod response) genes and their surrounding DNA sequences. - Theor. Appl. Genet. 125: 999-1014, 2012.

Tamura, K., Peterson, D., Peterson, N., Stecher, G., Nei, M., Kumar, S.: MEGA5: molecular evolutionary genetics analysis using maximum likelihood, evolutionary distance, and maximum parsimony methods. - Mol. Biol. Evol. 28: 27312739,2011

Tang, Y., Kang, H.Y., Tang, L., Diao, C.D., Li, D.Y., Zhu, W., Fan, X., Wang, Y., Zeng, J., Xu, L.L., Sha, L.N., Yu, X.F., Zhang, H.Q., Zhou, Y.H.: Phylogenetic analysis of tetraploid wheat based on nuclear DMC1 gene. - Biochem. Syst. Ecol. 70: 239-246, 2017.

Tang, Z.X., Yang, Z.J., Fu, S.L.: Oligonucleotides replacing the roles of repetitive sequences pAs1, pSc119.2, pTa-535, pTa71, CCS1, and pAWRC.1 for FISH analysis. - J. appl. Genet. 55: 313-318, 2014.

Thompson, J.D., Plewniak, F., Poch, O.: A comprehensive comparison of multiple sequence alignment programs. - Nucl. Acids Res. 27: 2682-2690, 1999.

Uauy, C., Distelfeld, A., Fahima, T., Blechl, A., Dubcovsky, J.: A $N A C$ gene regulating senescence improves grains protein, zinc, and iron content in wheat. - Science 314: 1298-1301, 2006.

Van Slageren, M.W.: Wild wheats: a monograph of Aegilops L. and Amblyopyrum (Jaub. \& Spach) Eig (Poaceae): a revision of all taxa closely related to wheat, excluding wild Triticum species, with notes on other genera in the tribe Triticcae, especially Triticum. - Dissertations \& Theses, Wageningen Agricultural University, Wageningen 1994.

Yamane, K., Kawahara, T.: Intra- and interspecific phylogenetic relationships among diploid Triticum-Aegilops species (Poaceae) based on base-pair substitutions, indels, and microsatellites in chloroplast noncoding sequences. - Amer. J. Bot. 92: 1887-1898, 2005.

Zaharieva, M., Ayana, N.G., Hakimi, A.A., Misra, S.C., Monneveux, P.: Cultivated emmer wheat (Triticum dicoccon Schrank), an old crop with promising future: a review. - Genet. Resour. Crop Evol. 57: 937-962, 2010.

Zohary, D., Hopf, M.: Domestication of Plants in the Old World: the Origin and Spread of Cultivated Plants in West Asia, Europe and the Nile Valley. - Oxford University Press, Oxford 2000. 